\title{
Epitaxial Growth of Optoelectronically Active Ga(As)Sb Quantum Dots on Al-Rich AlGaAs with GaAs Capsule Layers
}

\author{
Johannes Strassner $\mathbb{D},{ }^{1}$ Johannes Richter, ${ }^{1}$ Thomas Loeber $\mathbb{D}^{2},{ }^{2}$ Christoph Doering $\mathbb{D}{ }^{1}$ \\ and Henning Fouckhardt $\mathbb{D}^{1}$ \\ ${ }^{1}$ Integrated Optoelectronics and Microoptics Research Group, Physics Department, Technische Universität Kaiserslautern (TUK), \\ PO Box 3049, D-67653 Kaiserslautern, Germany \\ ${ }^{2}$ Nanostructuring Center (NSC), Physics Department, Technische Universität Kaiserslautern (TUK), PO Box 3049, \\ D-67653 Kaiserslautern, Germany
}

Correspondence should be addressed to Johannes Strassner; strassner@physik.uni-kl.de

Received 11 September 2020; Revised 6 April 2021; Accepted 30 April 2021; Published 19 May 2021

Academic Editor: Miguel Navarro-Cia

Copyright (c) 2021 Johannes Strassner et al. This is an open access article distributed under the Creative Commons Attribution License, which permits unrestricted use, distribution, and reproduction in any medium, provided the original work is properly cited.

\begin{abstract}
We present a study of optoelectronically active $\mathrm{Ga}(\mathrm{As}) \mathrm{As}$ quantum dots (QDs) on Al-rich $\mathrm{Al}_{x} \mathrm{Ga}_{1-x} \mathrm{As}$ layers with $\mathrm{Al}$ concentrations up to $x=90 \%$. So far, however, it has not been possible to grow optoelectronically active Ga(As)As QDs epitaxially directly on and in between Al-rich barrier layers in the AlGaInAsSb material system. A QD morphology might appear on the growth front, but the QD-like entities will not luminesce. Here, we use photoluminescence (PL) measurements to show that thin Al-free capsule layers between Al-rich barrier layers and the QD layers can solve this problem; this way, the QDs become optoelectronically active; that is, the dots become QDs. We consider antimonide QDs, that is, Ga(As)Sb QDs, either on GaAs for comparison or on $\mathrm{Al}_{x} \mathrm{Ga}_{1-x}$ As barriers $(x>10 \%)$ with GaAs capsule layers in between. We also discuss the influence of QD coupling both due to stress/strain from neighboring QDs and quantum-mechanically on the wavelength of the photoluminescence peak. Due to their mere existence, the capsule layers alter the barriers by becoming part of them. Quantum dots applications such as QD semiconductor lasers for spectroscopy or QDs as binary storage cells will profit from this additional degree of design freedom.
\end{abstract}

\section{Introduction}

Inorganic quantum dots (QDs), especially concerning their potential use in semiconductor lasers, have been in the research focus for 25 years now $[1,2]$. In particular, Ga(As) $\mathrm{Sb}$ quantum dots (QDs) grown on GaAs [3-13] are of interest as an active material for efficient lasers in the near- and midinfrared. These lasers could, for example, be used for sensitive spectroscopy or even long-distance optical communications via glass fibers [11]. On account of the strong hole confinement and (due to a type-II band alignment) no electron confinement, each QD might also be suitable for use as a binary storage cell in a quantum dot memory device $[11,14-16]$.
On the one hand, to increase the degree of design freedom, in principle, the QDs could also be grown onto an Al-rich $\mathrm{Al}_{x} \mathrm{Ga}_{1-x}$ As layer (with $x>10 \%$ ) as the barrier layer. In the case of antimonide QD applications in semiconductor lasers, this would potentially increase the range of available emission wavelengths. In the case of antimonide QDs as binary storage cells, this approach would raise the confinement energy $C$, but it would also result in a transition from a type-II to a type-I band alignment. This means that not only the holes but also the electrons will be confined, such that-by trend - the carrier lifetime will be reduced due to an increased rate of electron-hole recombination.

On the other hand, the biggest challenge in using Al-rich barrier layers for $\mathrm{Ga}(\mathrm{As}) \mathrm{Sb} \mathrm{QDs}$ is the fact that they are 
usually not optoelectronically active [17, 18], although QDlike objects might evolve at these barriers during Stranski-Krastanov growth (SK); see Figure 1.

This has been attributed to crystal defects at the QDs' bases, which lead to mismatch dislocations, which dislocate the whole QDs by $1 \mathrm{ML}$ [19]. The resulting stress/strain cannot relax, especially in the case of Al-rich surrounding material.

Thus, despite their promising potential applications, the growth of active antimonide QDs at and in between Al-rich barriers with strong luminescence does not seem to be feasible.

In this contribution, we show that the thin, so-called "capsule layers" made from GaAs between the QD layers and the original Al-rich barriers can solve this problem.

In this case, each capsule layer has to be considered part of a new (double-layer) barrier. With that, however, the confinement energy and the photon energy of the luminescence emission might be altered to some extent. In the case of antimonide QD semiconductor lasers, this varies the range of possible emission wavelengths. For the use of QDs as binary storage cells, two aspects have to be considered: either type-II band alignment can be maintained. At the same time, the hole lifetime can even be increased further, or the band alignment inadvertently changes to type-I, making electron-hole recombination more likely and thus reducing the carrier lifetime.

\section{Antimonide QD Growth}

Each sample is grown on an undoped (001) GaAs substrate with an R450 molecular beam epitaxy (MBE) machine from DCA Oy, Finland. The beam equivalent pressure of Ga differs slightly from sample to sample, that is, between 1.6 and $1.8 \cdot 10^{-7} \mathrm{hPa}$. The $\mathrm{Sb} / \mathrm{Ga}$ (V/III) flux (or partial pressure) ratio is adjusted with a valved $\mathrm{Sb}$ cracker from ADDON, Carrières-Sur-Seine, France. Wafers are bought "epi-ready" from Wafer Technology Ltd., Milton Keynes, UK.

For each sample, first, a $200 \mathrm{~nm}$ thick GaAs buffer layer is grown on the substrate with $0.3 \mathrm{ML} / \mathrm{s}$ (monolayer per second) at a GaAs substrate temperature of $630^{\circ} \mathrm{C}$, the latter measured with a pyrometer. Then, the sample is cooled down to the chosen quantum dot growth temperature, that is, to $530^{\circ} \mathrm{C}$ for all cases shown here. The GaSb material is deposited on an As-rich surface with a nominal QD growth rate of also $0.3 \mathrm{ML} / \mathrm{s}$.

The Stranski-Krastanov (SK) growth of binary GaSb QDs on GaAs would be associated with a $7.8 \%$ lattice mismatch. Due to As and Sb intermixing (resulting in GaAsSb QDs) [20, 21], the growth of these QDs is more complex than that of InAs QDs on GaAs. The epitaxial process depends on the exact control of the $\mathrm{Sb} / \mathrm{Ga}$ (V/III) flux ratio, the growth temperature, and the nominal coverage $[16,18,22]$.

$\mathrm{An}$ increased $\mathrm{Sb} / \mathrm{Ga}$ ratio results in a red-shift of the luminescence or laser emission due to lower As content in the QDs and the size of the QDs, which tend to get larger. However, an increase in the growth temperature for a constant $\mathrm{Sb} / \mathrm{Ga}$ ratio leads to a blue-shift due to stronger $\mathrm{As}$ in-diffusion into the QDs. This intermixing of Sb and As dominates the luminescence peak shift; that is, the corresponding blue-shift usually overcompensates for the redshift of larger dots.

Thus, for example, to achieve a maximum emission wavelength, the As content within $\mathrm{Ga}(\mathrm{As}) \mathrm{Sb}$ QDs has to be as low as possible. Other authors introduced pre- and postsoaking steps with Sb before and after QD growth [23]. In work leading to $[11,16,18]$, we successfully achieved strong photoluminescence (PL) emission from QDs with a wavelength peak at $1.309 \mu \mathrm{m}$. To do so, we applied a growth protocol with a $2: 1 \mathrm{Sb} / \mathrm{Ga}$ flux ratio, a substrate growth temperature of $530^{\circ} \mathrm{C}$, and a nominal coverage of the growth front of $2 \mathrm{ML}$. Furthermore, we interrupted the QD growth for $10 \mathrm{~s}$ while keeping the $\mathrm{Sb}$ flux turned on after the deposition of the QD material as a long postsoaking step. This growth interruption stabilizes the surface and suppresses As/Sb intermixing to some extent [11]. With this optimized growth protocol, we could obtain GaAsSb QDs with an As content of only around $30 \%$ and $\mathrm{Sb}$ content maximized to about $70 \%$ $[16,18]$. This protocol is mentioned here exemplarily to report the growth parameters which are optimal for longwavelength emission. For the results described in this contribution, slightly modified growth protocols have been applied.

\section{Antimonide QD Design}

The design of QDs also includes the design of the barriers, by which the dots become quantum dots, that is, threedimensional potential wells. Figure 2 reveals the energy level/band schemes (under flat band conditions) for three different barrier materials and a hypothetical pure $\mathrm{GaSb}$ QD in each case, that is, an antimonide QD without any As contents. These values have been calculated with the nextnano++ software by nexnano $\mathrm{GmbH}$, Poing, Germany, which will be explained in some more detail below. The valence band offset $D=C+L$ of the bulk material for the hole QDs can be divided into the confinement energy $C$ (energy difference of the hole state in the QD and the bulk valence band edge of the material used for the QDs) and the localization energy $L$ (energy difference of the hole state in the QD and the potential barrier edge). The larger the Al content in the barriers, the larger both $D$ and $L$-assuming the same QD size and shape, that is, for our calculations: pyramidal QDs with a base width of $25 \mathrm{~nm}$ and a height of $3 \mathrm{~nm}$.

Since it does not seem possible to grow optoelectronically active antimonide $(\mathrm{Ga}(\mathrm{As}) \mathrm{Sb})$ QDs on $\mathrm{Al}_{x} \mathrm{Ga}_{1-x} \mathrm{As}$ layers with high $\mathrm{Al}$-contents $(x>10 \%)$, here we suggest capsule layers in between the Al-rich (formerly single-layer) barrier and the pure QD layer. These capsules to the QDs are designed to be made from pure GaAs and to have a thickness of 5-20 nm. To protect the $\mathrm{Al}_{x} \mathrm{Ga}_{1-x}$ As layers (especially with high $\mathrm{Al}$ contents) from oxidation, the surfaces can be passivated to ensure the long-term stability of the components [24-27]. 


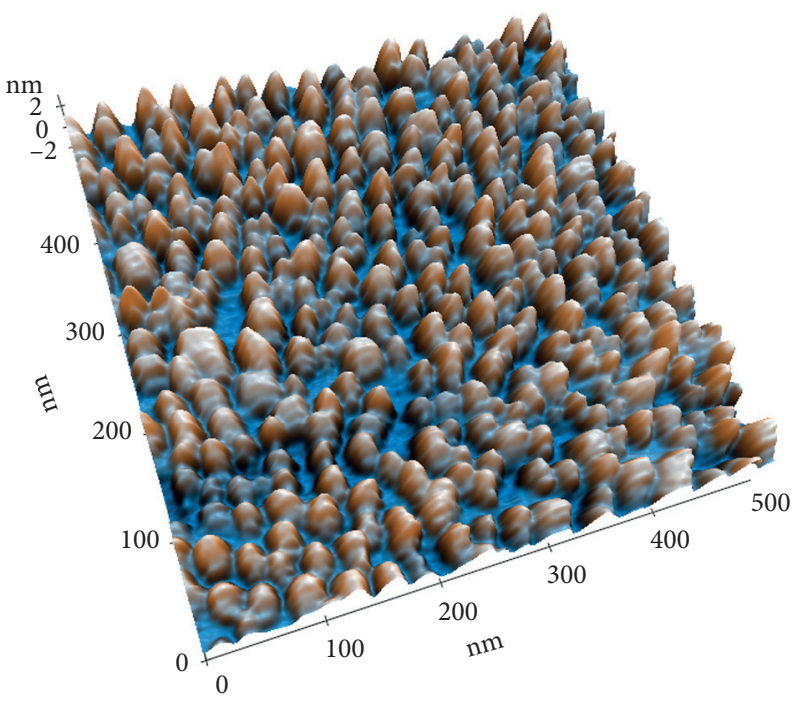

(a)

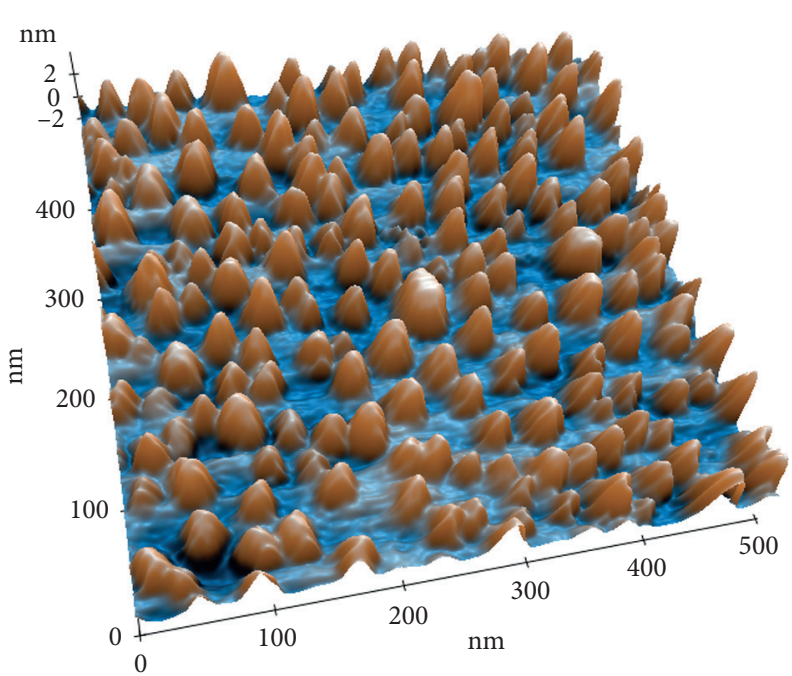

(b)

Figure 1: Atomic force micrographs of (a) active Ga(As)Sb QDs on GaAs and (b) nonactive QD-like objects grown from Ga(As)Sb on $\mathrm{Al}_{0.5} \mathrm{Ga}_{0.5} \mathrm{As}$. Be aware of the different scale in the height dimension as opposed to both lateral dimensions along the growth front.

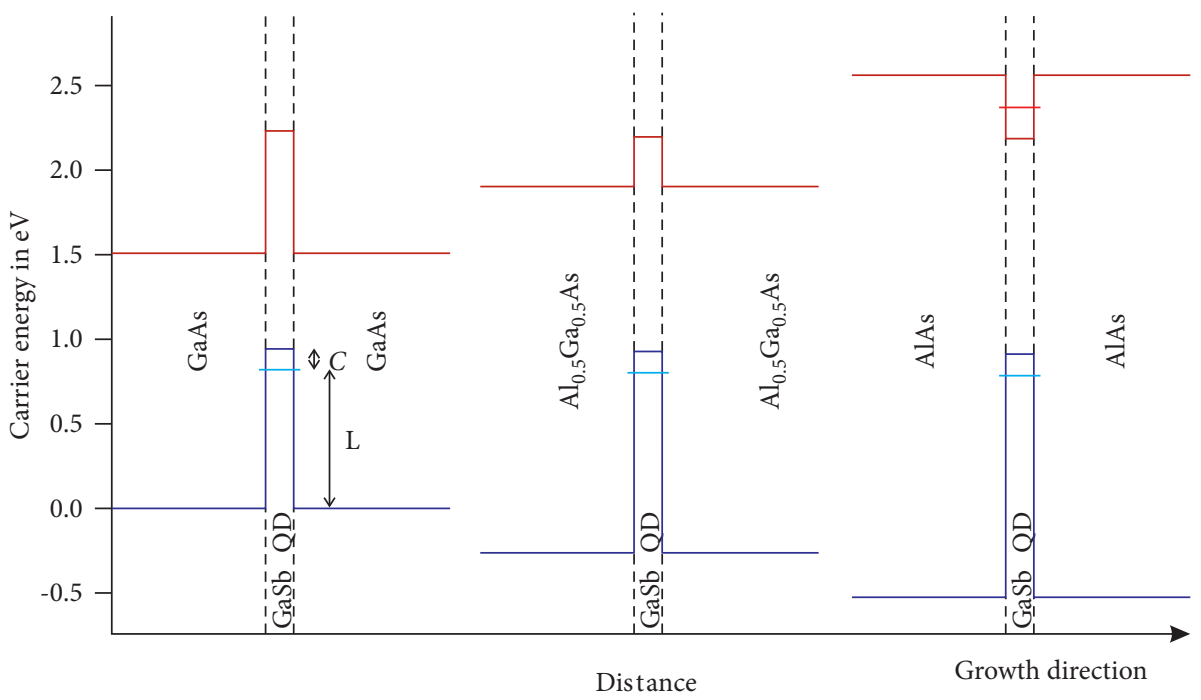

FIGURE 2: Electronic/hole QD energies and bulk material band edges for three QD heterostructures with different Al contents $x$ in the singlelayer barriers (flat band conditions including stress/strain). Pyramidal QDs with $25 \mathrm{~nm}$ base width and $3 \mathrm{~nm}$ height are assumed.

\section{Numerical Results on QD Coupling}

There are two main reasons for the coupling of QDs, both "horizontally" (within a QD layer) and "vertically" (from one QD layer to the next): mechanical stress/strain exerted from neighboring QDs and quantum-mechanical coupling due to the overlap of the wave functions.

To get an understanding (before investigating doublelayer barriers) on how strongly both of these reasons for coupling affect the spectral luminescence peak position, we have pursued numerical calculations again using the nextnano++ software by nextnano $\mathrm{GmbH}$, Poing, Germany, for the case of pure GaAs (single-layer) barriers. The program is based on an 8-band $\mathbf{k} \cdot \mathbf{p}$ algorithm [28, 29].
Mechanical stress/strain is automatically considered in the program, but stress relaxation by the evolution of crystal defects (one-dimensional or dislocations) is not because specific positions of the atoms corresponding to the $\alpha$ zinc blende crystal structure are assumed.

4.1. Scheme of Numerical Calculations. The nextnano++ software, which is based on a perturbation-theoretical approach, is useful to determine the quantum-mechanical eigenfunctions and eigenstates of the charge carriers, for example, within QDs. The solutions are developed locally around and at any specific point in momentum space, for example, at the $\Gamma$ point (as we do here). The nextnano++ 
software takes care of any mechanical stress/strain and the localization of the carriers in all three dimensions in the QDs.

Physical parameters, such as temperature, have to be chosen, as well as the model to describe the lattice stress/ strain.

A three-dimensional spatial mesh is defined. For each point of the mesh, the material composition has to be chosen. Schroedinger's and Poisson's equations are solved iteratively until a self-consistent solution is reached (if at all). For different spatial areas, the software user can determine whether the calculation should be performed considering quantum-mechanics (i.e., Schroedinger and Poisson) or classically (i.e., Poisson) only. In the first case, both stress/ strain and quantum-mechanical coupling will be taken into account; in the second case, coupling via stress/strain only will be taken into account.

For the calculations, we assume an orderly $3 \times 3$ array of QDs arranged in a square in all of the QD layers.

When quantum-mechanical effects need to be considered, the volume of the quantum dot itself and the volume around it for a distance of up to $6 \mathrm{~nm}$ in all six directions from the outer edges (or the tip) of the quantum dot are included. Calculations for distances of more than $6 \mathrm{~nm}$, however, would result in a tremendous increase in computing time.

This approach means that both the coupling via stress/ strain and the quantum-mechanical coupling is taken into account as long as distances between the QDs of less than $6 \mathrm{~nm}$ are assumed. For larger QD distances, however, which resemble the situation of our grown samples more closely, the calculations only reflect the influence of stress/strain on the carriers' energy levels.

4.2. In-Plane Coupling. For the calculations, we assume $\mathrm{GaAs}_{y} \mathrm{Sb}_{1-y}$ QDs of pyramidal shape with a base width of $25 \mathrm{~nm}$, a height of $3 \mathrm{~nm}$, and an Sb content of $(1-y)=0.7$.

A square-shaped $3 \times 3$ array of these $\mathrm{GaAs}_{0.3} \mathrm{Sb}_{0.7} \mathrm{QDs}$ is assumed for each calculation. Their "horizontal distance" is defined as the geometrical distance between the edges of any two nondiagonally neighboring QDs. For example, for a nominal distance of $12 \mathrm{~nm}$, the tips of two such QDs are $[(25 / 2)+12+(25 / 2)] \mathrm{nm}=37 \mathrm{~nm}$ apart. Our calculations are done for nominal horizontal QD distances of $1 \mathrm{~nm}, 2 \mathrm{~nm}$, $12 \mathrm{~nm}$, and $27 \mathrm{~nm}$.

The results are summarized in Figure 3. The quantities $\Delta E_{c}, \Delta E_{h h}$, and $\Delta E_{s o}$ denote the shift of the bulk conduction band edge, the bulk valence band edge for heavy holes $(h h)$, and that of the bulk valence band edge for split-off holes ( $s o$ ), respectively.

The differences in energy shifts for the $3 \times 3$ QDs are negligible on this scale, and only the mean values are given here. For the luminescence emission wavelength shift $\Delta \lambda$, the differences in the results for the nine QDs are larger so that the range is given here via the light-red shaded area. Differences between the values for the central QD and the four QDs at the corners of the $3 \times 3$ array/square and the four QDs in the middle of the square sides should be expected from the geometrically different coupling to adjacent QDs. However, even within any of these QD classes (e.g., QDs at the corners of the square), there are slight differences, which are related to the way the nextnano++ software algorithm computes the spatial mesh.

It is obvious from Figure 3 that there is a nonnegligible luminescence emission wavelength shift due to horizontal coupling via stress/strain as well as quantum-mechanically. For nominal horizontal QD distances of some nanometers, the wavelength shift can even exceed values of $50 \mathrm{~nm}$. The shift is always positive and thus has the same qualitative impact as an increase in the size of the QD or a reduction in As content. For horizontal QD distances $>6 \mathrm{~nm}$ (per definition of the calculation conditions) only stress/strain is incorporated for the calculated wavelength shift, which can still exceed tens of nanometers.

4.3. Cross-Plane Coupling. In Figure 4, the results of the calculations for an additional vertical coupling of the $\mathrm{GaAs}_{0.3} \mathrm{Sb}_{0.7}$ QDs are shown. "Additional" means that the values given in the diagram are stated relative to the "new" levels, that is, already shifted due to horizontal coupling according to Figure 3 . The calculations are pursued by the numerical implementation of two $3 \times 3$ square arrays of the same orientation on top of each other. The nominal horizontal distance between QDs is kept constant at $2 \mathrm{~nm}$ within both $3 \times 3$ arrays, in equivalence to a situation with QDs situated very close to each other.

The mean energy shifts for the two central QDs in the two $3 \times 3$ arrays are given in Figure 4 since they resemble the more realistic situation where others surround every QD. The fact that there are differences between the results for the two central QDs has to be attributed to the direction of working through the mesh by the software algorithm.

For these calculations, the nominal vertical distance of QDs on top of each other has been varied. This distance is defined by the one between the tip of the QDs in the "lower" QD layer and the base of the QDs in the "upper" QD layer. The values, which have been assumed for the calculations, are $2 \mathrm{~nm}, 22 \mathrm{~nm}$, and $47 \mathrm{~nm}$, respectively.

For the heavy hole $(h h)$ and the split-off hole (so) energy level shifts, the differences for both central QDs are negligible. For the bulk conduction band edge shift, the differences are not negligible, but for the sake of readability of the diagram, only the mean values are shown in Figure 4 together with the mean values for the wavelength shifts $\Delta \lambda$.

As can be deduced from this figure, vertical coupling also leads to considerable (positive-valued) luminescence emission wavelength shifts. For small vertical QD distances of a few nanometers, the additional shift comes close to shifts due to the horizontal interaction; that is, it reaches values of around $40 \mathrm{~nm}$. Therefore, for small QD distances, the overall wavelength shift due to both in- and cross-plane coupling (via mechanical stress/strain and quantum-mechanically) can show values of 80 to $90 \mathrm{~nm}$.

This (positive) wavelength shift value comes close to the (negative) value, which can be expected from Figure 5 according to $[16,18]$ for the case of uncoupled QDs with 


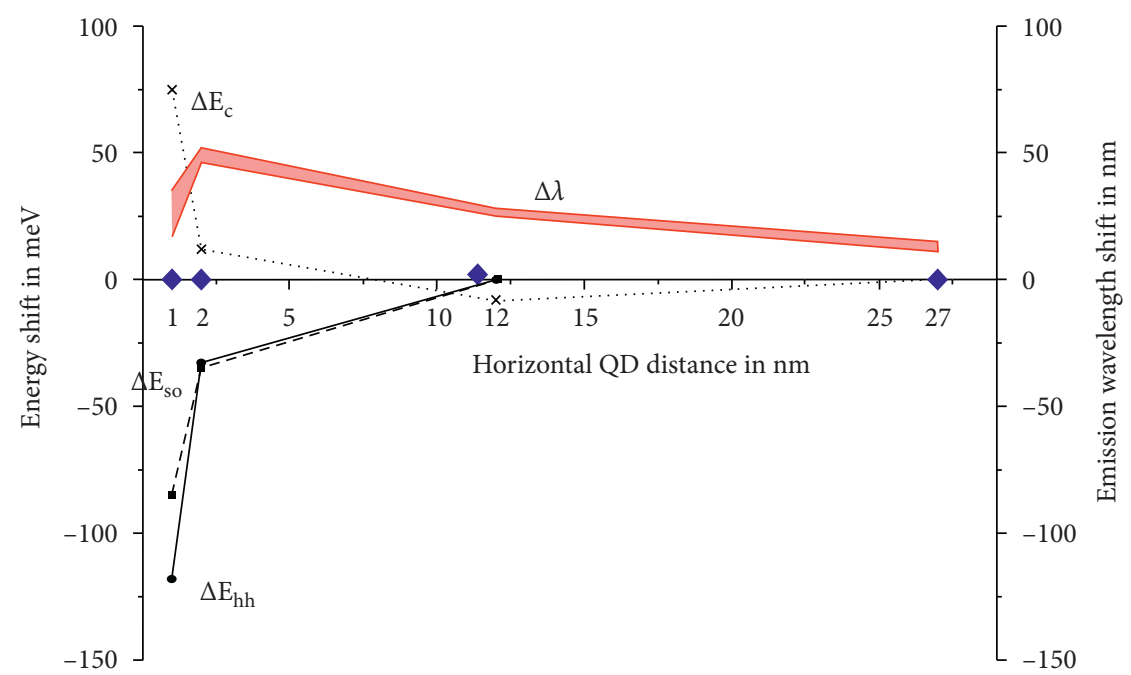

Figure 3: Numerical results on the impact of horizontal/in-plane coupling of $\mathrm{GaAs}_{0.3} \mathrm{Sb}_{0.7}$ QDs on GaAs arranged in a $3 \times 3$ square array, with an assumed horizontal QD distance of $1 \mathrm{~nm}, 2 \mathrm{~nm}, 12 \mathrm{~nm}$, or $27 \mathrm{~nm}$, respectively. The lines are only guides to the eye. The quantities $\Delta E_{c}$, $\Delta E_{h h}$, and $\Delta E_{s o}$ are the shifts of the bulk conduction $(c)$ band edge, the bulk valence band edge for heavy holes $(h h)$, and the bulk valence band edge for split-off holes (so), respectively. For the energy level shifts the mean values for the nine QDs are reported here. For the luminescence emission wavelength shift $\Delta \lambda$ a range is given via the light-red shaded area.

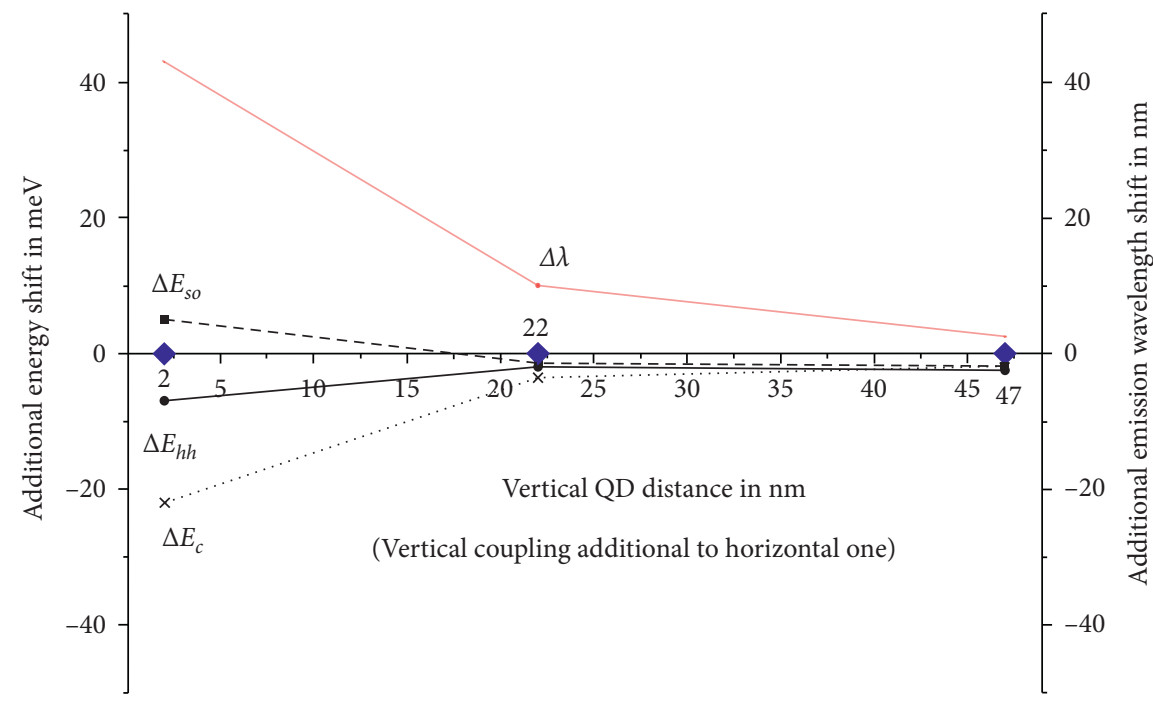

FIGURE 4: Numerical results on the impact of additional vertical/cross-plane coupling of $3 \times 3 \mathrm{GaAs}_{0.3} \mathrm{Sb}_{0.7}$ QDs on GaAs arranged in two $3 \times 3$ square arrays on top of each other with a nominal horizontal QD distance of $2 \mathrm{~nm}$. The nominal vertical QD distance is $2 \mathrm{~nm}$, $22 \mathrm{~nm}$, or $47 \mathrm{~nm}$, respectively. The lines are only guides to the eye. The quantities $\Delta E_{c} \Delta E_{h h}$, and $\Delta E_{s o}$ denote the energy shifts again, but now only the additional shifts due to vertical coupling. Mean values are given here. For the hh and the so hole energy level shifts, the differences for both central QDs are negligible anyway. But for the conduction band edge shift, the differences are not negligible. Also, the mean values for the wavelength shifts $\Delta \lambda$ are shown.

$25 \mathrm{~nm}$ base length and $3 \mathrm{~nm}$ height and a reduction of the Sb content in the QDs from $65 \%$ to $55 \%$, as calculated with nextnano++.

For larger QD distances, where only coupling by stress/ strain is taken into account, but not quantum-mechanical coupling, the wavelength shift can still reach up to $25 \mathrm{~nm}$, which is in good quantitative agreement with the experimental results further below.

\section{Photoluminescence (PL) Results}

For comparison, to gain some understanding of the influence of the GaAs capsule layers, we experimentally investigate eight different samples with $4 \mathrm{Ga}(\mathrm{As}) \mathrm{Sb}$ QD layers and pure GaAs (Al-free single-layer) barriers in between. For any one of the eight samples, the barrier thickness is kept constant. The barrier thickness is varied, from 10 to $80 \mathrm{~nm}$ in 


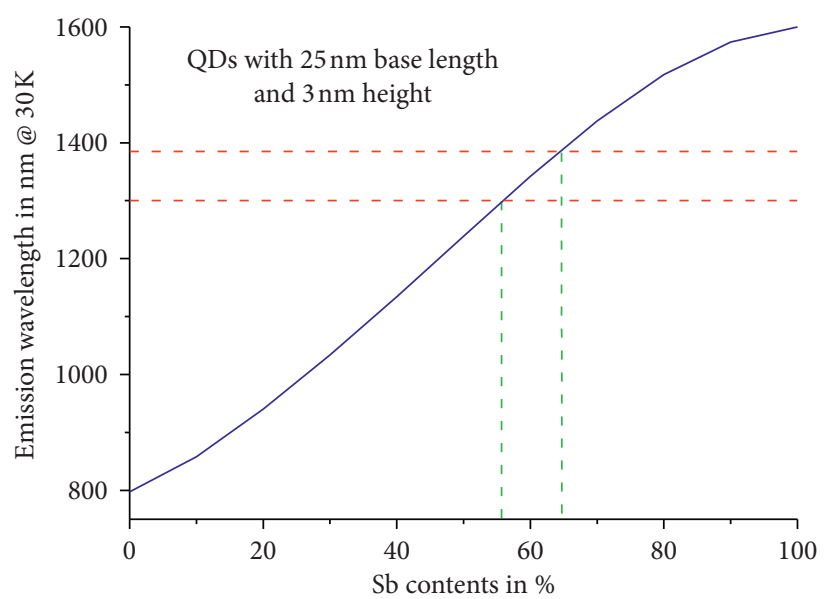

FIGURE 5: Simulated emission wavelength for different uncoupled QD sizes and Sb contents $(1-y)$ within the GaAs $\mathrm{Sb}_{1-y} \mathrm{QDs}_{\mathrm{s}}$ on GaAs substrate. The wavelength is above $1.3 \mu \mathrm{m}$ for $\mathrm{Sb}$ contents of $55 \%$ and more.

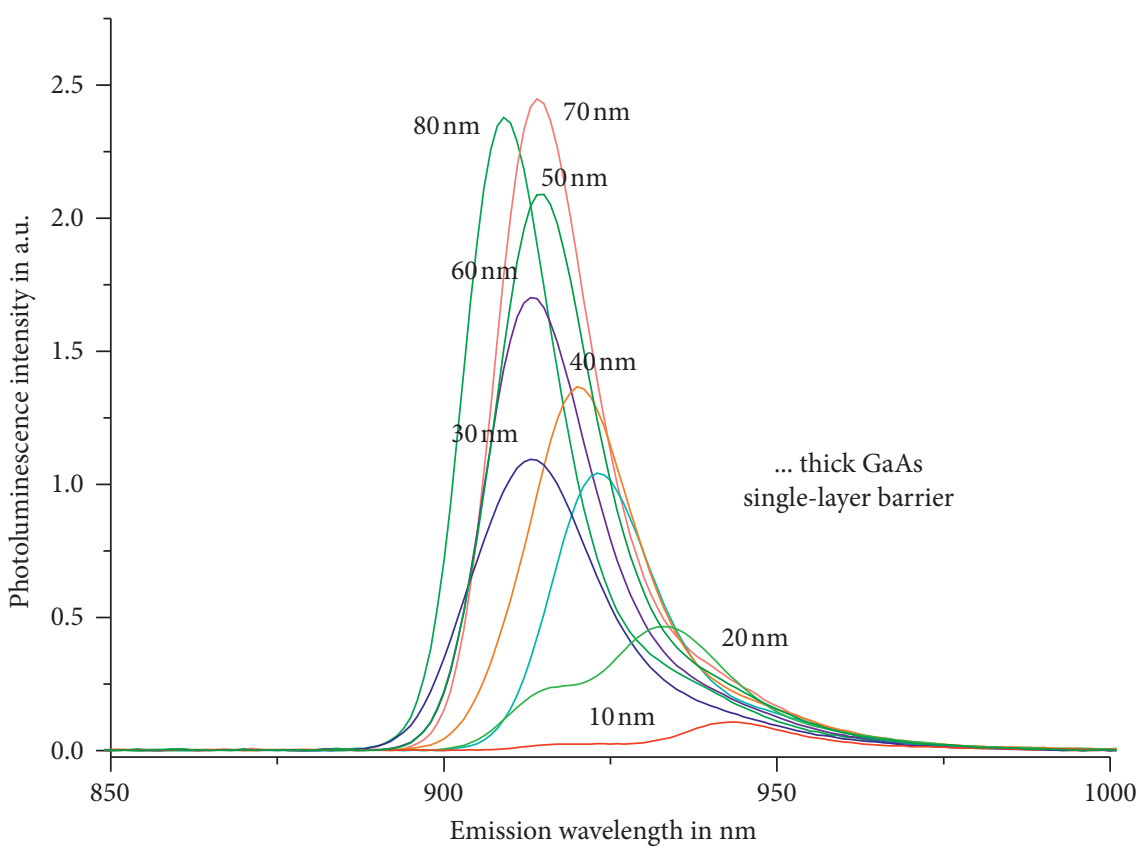

FIGURE 6: Results of photoluminescence (PL) measurements for different samples, each with 4 QD layers, but different GaAs single-layer barrier thickness. The QD layers have been grown with a V/III flux ratio of $1: 1$, a substrate growth temperature of $530^{\circ} \mathrm{C}$, and a nominal coverage of $3 \mathrm{ML}$. The base width of these QDs is $25 \mathrm{~nm}$, and their height is $3 \mathrm{~nm}$. For typical As-content, refer to Figure 5.

steps of $10 \mathrm{~nm}$. The base width of these QDs is $25 \mathrm{~nm}$, and their height is $3 \mathrm{~nm}$.

Figure 6 reveals experimental photoluminescence (PL) results for these eight samples. Although there are growth tolerances, which cause unexpected deviations from the general trend (e.g., the PL peak intensity for a barrier thickness of $60 \mathrm{~nm}$ falls short), there is evidence that coupling is weak for barrier thicknesses $>50 \mathrm{~nm}$, where the PL peak positions and heights do not change strongly anymore.

The value of $50 \mathrm{~nm}$ barrier thickness gives an approximate lower limit for the single-layer barrier thickness desirable for growth processes. For double-layer barriers, the height and thickness of the GaAs capsule layers have to be considered, too.

Therefore, we start to use double-layer barriers made from $\mathrm{Al}_{x} \mathrm{Ga}_{1-x}$ As on the far side of any QD layer and the GaAs capsule layer adjacent to any QD layer. So in between any two of the 4 QD layers (grown as before), there are now two GaAs capsule layers (one from each QD layer) and one $\mathrm{Al}_{x} \mathrm{Ga}_{1-x}$ As layer in between. A corresponding energy band diagram for $\mathrm{Al}_{0.9} \mathrm{Ga}_{0.1}$ As layers is shown in Figure 7 .

In a first test run, we use $20 \mathrm{~nm}$ thick GaAs capsule layers and $\mathrm{Al}_{0.5} \mathrm{Ga}_{0.5}$ As layers $(x=0.50)$ with varying thicknesses from sample to sample, that is, $10 \mathrm{~nm}, 30 \mathrm{~nm}$, 


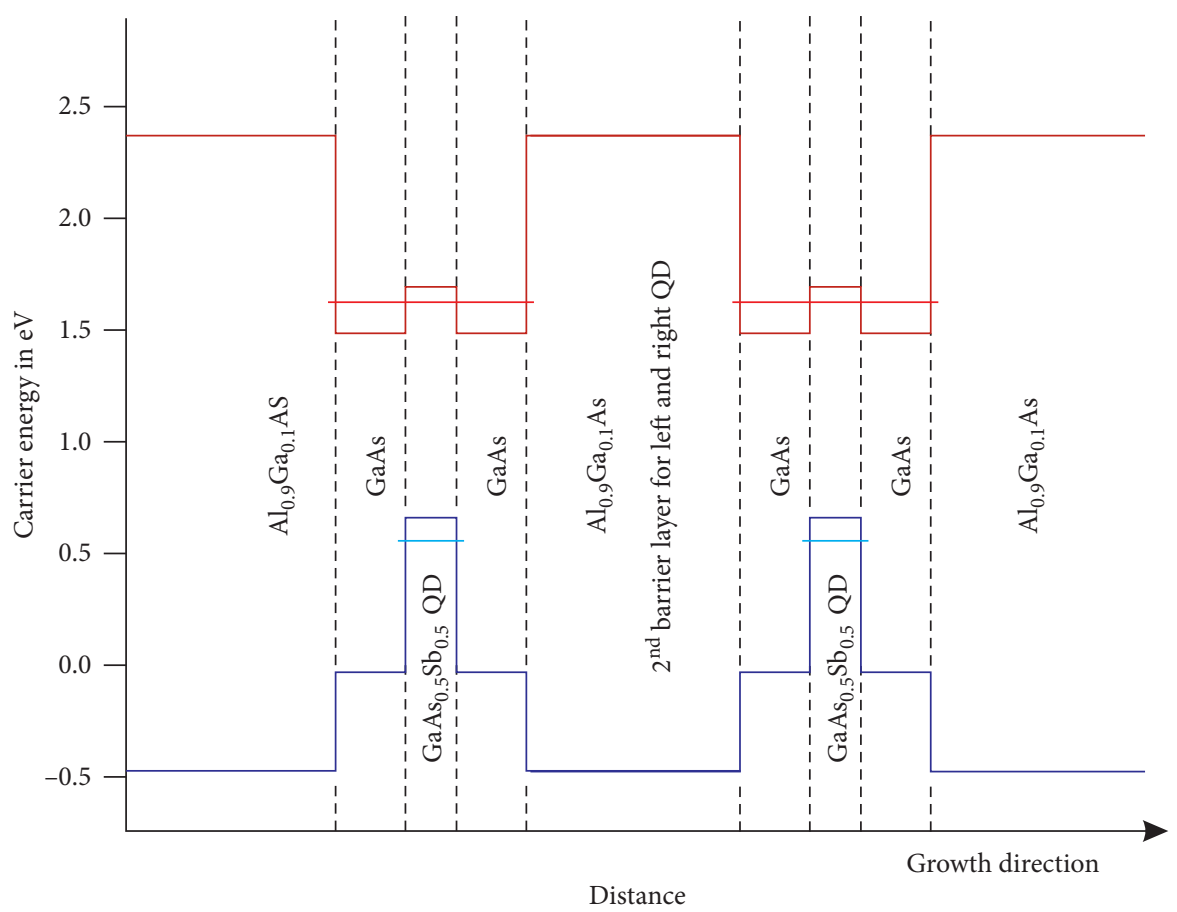

Figure 7: Energy level/band diagram of a GaAs ${ }_{0.5} \mathrm{Sb}_{0.5}$ QD in between pure GaAs capsule layers, and those in between $\mathrm{Al}_{0.9} \mathrm{Ga}_{0.1} \mathrm{As}$ layers (flat band conditions including stress/strain). Now electrons have a bound state due to the GaAs-layers. The capsule layers are assumed to have a thickness of $20 \mathrm{~nm}$ each. For the QDs a pyramidal shape and a base width of $25 \mathrm{~nm}$ and a height of $3 \mathrm{~nm}$ are assumed.

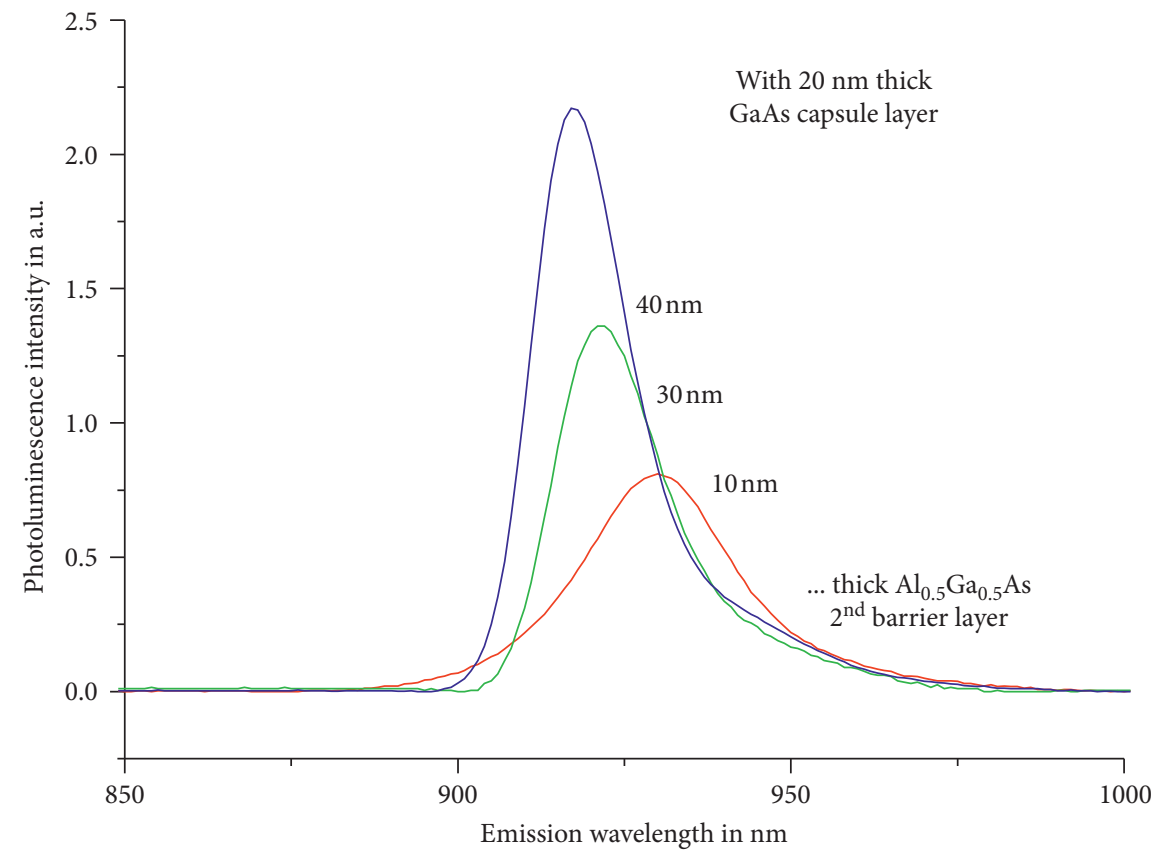

FIGURE 8: Results of photoluminescence (PL) measurements for three different samples, each with 4 QD layers, but double-layer barriers. Each barrier is made from a $20 \mathrm{~nm}$ thick GaAs capsule layer and an $\mathrm{Al}_{0.5} \mathrm{Ga}_{0.5}$ As layer of either one of three possible thicknesses, that is, $10 \mathrm{~nm}, 30 \mathrm{~nm}$, and $40 \mathrm{~nm}$. The QD layers have been grown as described for the samples shown in Figure 6.

and $40 \mathrm{~nm}$. The PL results are given in Figure 8. The results are similar to those in Figure 6 for the case of single-layer barriers. If the $\mathrm{Al}_{0.5} \mathrm{Ga}_{0.5}$ As layers are too thin $(<40 \mathrm{~nm})$, stress/strain cannot be relieved from one QD layer to the next one, and the PL intensity is weak. Thus, from now on, we choose $\mathrm{Al}_{x} \mathrm{Ga}_{1-x}$ As layers of a minimum thickness of $40 \mathrm{~nm}$.

Next, we employ three samples with 4 QD layers (grown as before) and $40 \mathrm{~nm}$ thick $\mathrm{Al}_{0.5} \mathrm{Ga}_{0.5}$ As layers as parts of the double-layer barriers. The GaAs capsule layers are 5, 10, and 


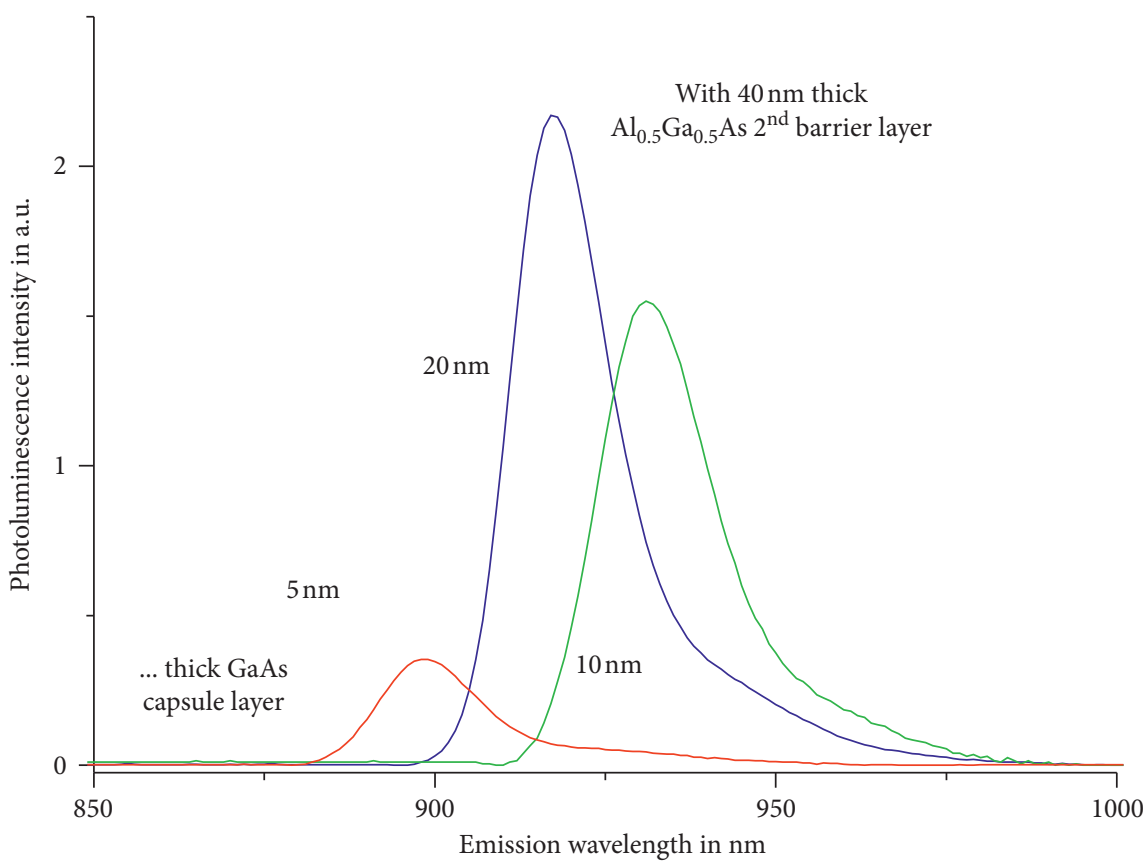

FIGURE 9: Results of photoluminescence (PL) measurements for three different samples, each with 4 QD layers, but double-layer barriers. Each barrier is made from a $5 \mathrm{~nm}, 10 \mathrm{~nm}$, and $20 \mathrm{~nm}$ thick GaAs capsule layer, respectively, and a $40 \mathrm{~nm}$ thick $\mathrm{Al}_{0.5} \mathrm{Ga}_{0.5} \mathrm{As}_{\mathrm{s}}$ layer. The QD layers have been grown as described above; see Figure 6.

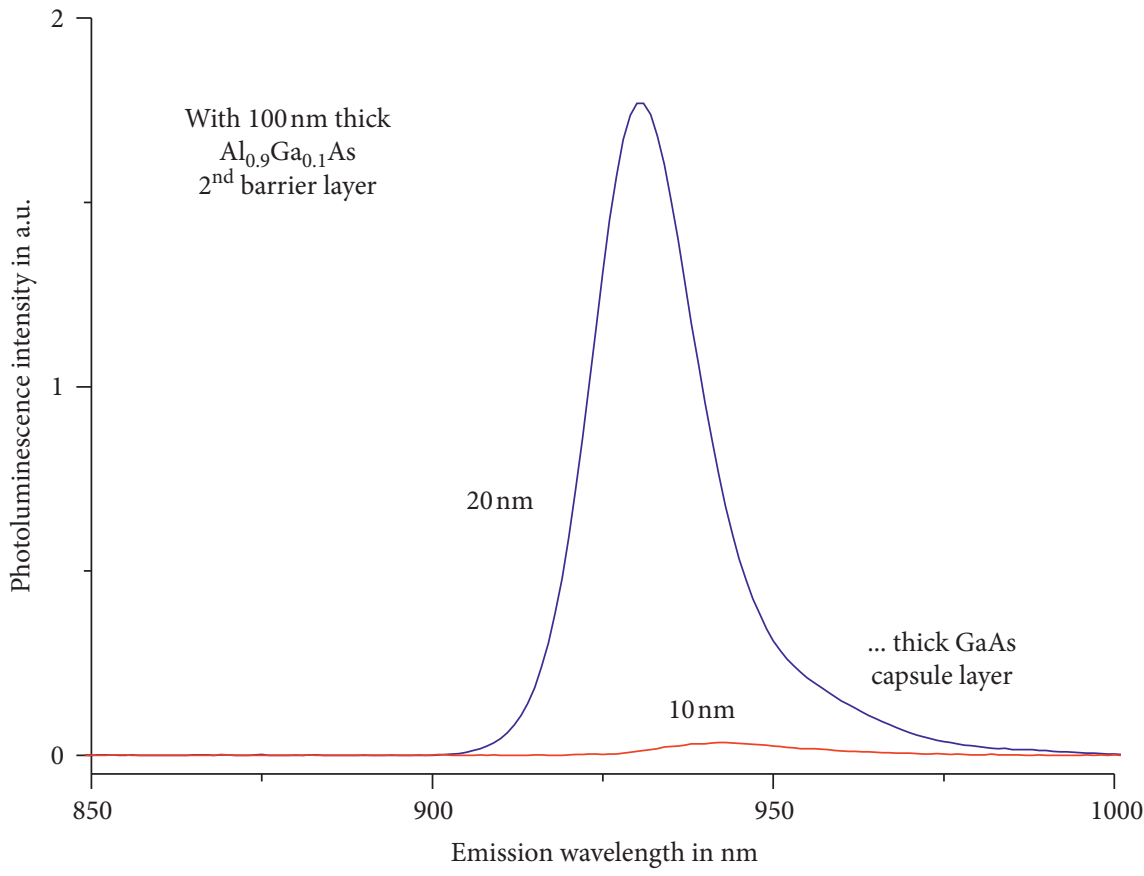

FIGURE 10: Results of photoluminescence (PL) measurements for two different samples, each with just 1 QD layer, but double-layer barriers. Each barrier is made from a $10 \mathrm{~nm}$ or $20 \mathrm{~nm}$ thick GaAs capsule layer, respectively, and an $\mathrm{Al}_{0.9} \mathrm{Ga}_{0.1}$ As $(x=0.9$ !) layer of a thickness of $100 \mathrm{~nm}$. The QD layer has been grown as described above.

$20 \mathrm{~nm}$ thick, respectively, for the three different samples. The photoluminescence results in Figure 9 reveal that a capsule thickness of $5 \mathrm{~nm}$ leads to a relatively low PL peak intensity. Obviously, for too thin GaAs capsule layers, the detrimental influence of the large interface energy of the $\mathrm{Al}_{0.5} \mathrm{Ga}_{0.5} \mathrm{As}$ layers is still too strong. We conclude that the GaAs capsule layer thickness should be at least $10 \mathrm{~nm}$, in the case of an $\mathrm{Al}_{0.5} \mathrm{Ga}_{0.5} \mathrm{As}$ layer as the Al-rich barrier part.

For the final sample run, we use a $100 \mathrm{~nm}$ thick $\mathrm{Al}_{0.9} \mathrm{Ga}_{0.1} \mathrm{As}$ (now $x=0.9$ !) barrier layer, in two samples with different GaAs capsule layer thicknesses of $10 \mathrm{~nm}$ and $20 \mathrm{~nm}$, respectively. The electronic/hole energy band scheme 
corresponds to that given in Figure 7, but now only 1 QD layer (grown as before) is used in these two samples. The PL results in Figure 10 reveal that now the capsule layers should rather have a thickness of $20 \mathrm{~nm}$ or more in order not to let the interface energy of the $\mathrm{Al}_{0.9} \mathrm{Ga}_{0.1}$ As layers deteriorate the optoelectronic activity of the QDs.

From the results in Figures 8 through 10, it can be deduced that a capsule layer will make the epitaxial growth of $\mathrm{Ga}(\mathrm{As}) \mathrm{Sb} \mathrm{QDs}$ “on” Al-rich barrier layers possible, if thick enough GaAs capsule layers are introduced, with each of the latter being part of a new (double-layer) barrier. For increasing the Al-contents of the Al-rich layers, the thickness of the capsule layers has to be increased to keep the QDs optoelectronically active.

\section{Conclusions}

To increase the degree of freedom for antimonide ( $\mathrm{Ga}$ (As) $\mathrm{Sb})$ quantum dot (QD) design, Al-rich $\mathrm{Al}_{x} \mathrm{Ga}_{1-x}$ As barriers would be helpful. Unfortunately, those QDs cannot be grown next to such Al-rich layers since the interface energy would make the QDs optoelectronically nonactive.

In this contribution, we have verified experimentally that pure GaAs capsule layers between the QD layer and the Alrich layers on both sides of the QD layers can solve this problem. The QDs will be optoelectronically active, as long as the capsule layers are thick enough, depending on the $\mathrm{Al}$ contents of the Al-rich barrier layers.

Each capsule layer then forms a part of any barrier; that is, each barrier is made up of two layers, which has to be taken into account for the QD design, for example, when aiming at a specific wavelength for the luminescence emission peak.

As our numerical calculations indicate, coupling of neighboring QDs via mechanical stress/strain and quantummechanical has to be taken into account in the numerical design since this can cause a spectral shift of the luminescence emission peak of several tens of nanometers. QD layer sequences as active material in semiconductor lasers are useful for applications where specific emission wavelengths are needed, such as infrared spectroscopy.

\section{Data Availability}

The data used to support the findings of this study are included within the article. Should further data or information be required, these are available from the corresponding author upon request.

\section{Conflicts of Interest}

The authors declare that there are no conflicts of interest regarding the publication of this paper.

\section{Acknowledgments}

This work was funded in the framework of the VIP project HOFUS of the German Federal Ministry of Education and Research (BMBF, Germany). Special thanks are due to nextnano $\mathrm{GmbH}$ for the simulation software and assistance.
The authors are grateful for some nextnano++ calculations by Jan Herbst of this research group. Also, technological assistance by the Nano Structuring Center (NSC) of the Technische Universität Kaiserslautern (TUK) is gratefully acknowledged.

\section{References}

[1] D. Bimberg, M. Grundmann, and N. N. Ledentsov, Quantum Dot Heterostructures, Wiley, Hoboken, NJ, USA, 1999.

[2] M. Grundmann, The Physics of Semiconductors: An Introduction Including Devices and Nanophysics, Springer, Berlin, Germany, 2002.

[3] F. Hatami, N. N. Ledentsov, M. Grundmann et al., "Radiative recombination in type-II GaSb/GaAs quantum dots," Applied Physics Letters, vol. 67, no. 5, pp. 656-658, 1995.

[4] E. R. Glaser, B. R. Bennett, B. V. Shanabrook, and R. Magno, "Photoluminescence studies of self-assembled InSb, GaSb, and AlSb quantum dot heterostructures," Applied Physics Letters, vol. 68, no. 25, pp. 3614-3616, 1996.

[5] F. Hatami, M. Grundmann, N. N. Ledentsov et al., "Carrier dynamics in type-II GaSb/GaAs quantum dots," Physical Review B, vol. 57, no. 8, pp. 4635-4641, 1998.

[6] T. Wang and A. Forchel, "Growth of self-organized GaSb islands on a GaAs surface by molecular beam epitaxy," Journal of Applied Physics, vol. 85, no. 5, pp. 2591-2594, 1999.

[7] L. Mueller-Kirsch, R. Heitz, U. W. Pohl et al., "Temporal evolution of $\mathrm{GaSb} / \mathrm{GaAs}$ quantum dot formation," Applied Physics Letters, vol. 79, no. 7, pp. 1027-1029, 2001.

[8] G. Balakrishnan, J. Tatebayashi, A. Khoshakhlagh et al., "III/V ratio based selectivity between strained Stranski-Krastanov and strain-free GaSb quantum dots on GaAs," Applied Physics Letters, vol. 89, no. 16, pp. 161104-1-161104-3, 2006.

[9] J. Tatebayashi, A. Khoshakhlagh, S. H. Huang, L. R. Dawson, G. Balakrishnan, and D. L. Huffaker, "Formation and optical characteristics of strain-relieved and densely stacked GaSb/ GaAs quantum dots," Applied Physics Letters, vol. 89, no. 20, pp. 203116-1-203116-3, 2006.

[10] J. Tatebayashi, A. Khoshakhlagh, S. H. Huang et al., "Lasing characteristics of $\mathrm{GaSb} / \mathrm{GaAs}$ self-assembled quantum dots embedded in an InGaAs quantum well," Applied Physics Letters, vol. 90, no. 26, pp. 261115-1-261115-3, 2007.

[11] J. Richter, J. Strassner, T. H. Loeber et al., "GaSb quantum dots on GaAs with high localization energy of $710 \mathrm{meV}$ and an emission wavelength of $1.3 \mu \mathrm{m}$," Journal of Crystal Growth, vol. 404, pp. 48-53, 2014.

[12] T. H. Loeber, D. Hoffmann, and H. Fouckhardt, "Dense lying self-organized GaAsSb quantum dots on GaAs for efficient lasers," Beilstein Journal of Nanotechnology, vol. 2, no. 1, pp. 333-338, 2011.

[13] T. H. Loeber, E. A. Hein, D. Hoffmann, C. Heisel, and H. Fouckhardt, "Generation of dense lying Ga(As)Sb quantum dots for efficient quantum dot lasers," Advanced Materials Research, vol. 684, pp. 285-289, 2013.

[14] M. Geller, C. Kapteyn, L. Müller-Kirsch, R. Heitz, and D. Bimberg, " $450 \mathrm{meV}$ hole localization in $\mathrm{GaSb} / \mathrm{GaAs}$ quantum dots," Applied Physics Letters, vol. 82, no. 16, pp. 2706-2708, 2003.

[15] A. Marent, "Development of a novel quantum dot storage cell,"Ph.D. Thesis (German), Department of Mathematics and Natural Sciences Technical, University Berlin, Berlin, Germany, 2011.

[16] J. Richter, "Optimized monitoring of antimonide growth of quantum dots and quantum dot surroundings with 
reflectance anisotropy spectroscopy," Ph.D. Thesis (German), Physics Department Technische, Universität Kaiserslautern(TUK), Verlag Dr. Hut, Kaiserslautern, Germany, 2014.

[17] T. H. Loeber, "Ga(As)Sb quantum dots and quantum dot lasers," Ph.D. Thesis (German), Physics Department Technische, Universität Kaiserslautern (TUK), Verlag Dr. Hut, Kaiserslautern, Germany, 2011.

[18] J. H. Strassner, "Epitaxial growth and optoelectronic properties of $\mathrm{Ga}(\mathrm{As}) \mathrm{Sb}$ quantum dots on $(\mathrm{Al}) \mathrm{GaAs}$ by variation of barriers," Ph.D. Thesis (German), Physics Department Technische, Universität Kaiserslautern (TUK), Verlag Dr. Hut, Kaiserslautern,Germany, 2016.

[19] E. P. Smakman, M. DeJarld, M. Luengo-KOvac et al., "Height stabilization of $\mathrm{GaSb} / \mathrm{GaAs}$ quantum dots by Al-rich capping," APL Materials, vol. 2, no. 9, pp. 096111-1-096111-7, 2014.

[20] Q. Xie, J. E. van Nostrand, J. L. Brown, and C. E. Stutz, "Arsenic for antimony exchange on $\mathrm{GaSb}$, its impacts on surface morphology, and interface structure," Journal of Applied Physics, vol. 86, no. 1, pp. 329-337, 1999.

[21] R. Timm, H. Eisele, A. Lenz et al., "Structure and intermixing of GaSb/GaAs quantum dots," Applied Physics Letters, vol. 85, no. 24 , pp. 5890-5892, 2004.

[22] J. Strassner, J. Richter, T. H. Loeber, and H. Fouckhardt, "Growth control of $\mathrm{Ga}(\mathrm{As}) \mathrm{Sb}$ quantum dots (QD) on GaAs with reflectance anisotropy spectroscopy (RAS)," Proceedings of SPIE, vol. 9288, pp. 92880F-1-92880F-8, 2014.

[23] J. Schmitz, J. Wagner, F. Fuchs, N. Herres, P. Koidl, and J. D. Ralston, "Optical and structural investigations of intermixing reactions at the interfaces of InAs/AlSb and InAs/ GaSb quantum wells grown by molecular-beam epitaxy," Journal of Crystal Growth, vol. 150, pp. 858-862, 1995.

[24] Y. Nannichi, J.-F. Fan, H. Oigawa1, and A. Koma, "A model to explain the effective passivation of the GaAs surface by $\left(\mathrm{NH}_{4}\right)_{2} \mathrm{~S}_{\mathrm{x}}$ treatment," Japanese Journal of Applied Physics, vol. 27, no. 12, pp. L2367-L2369, 1988.

[25] T. Ohno, "Sulfur passivation of GaAs surfaces," Physical Review B, vol. 44, no. 12, pp. 6306-6311, 1991.

[26] T. Tamanukil, F. Koyama, and K. Iga, "Ammonium sulfide passivation for $\mathrm{AlGaAs} / \mathrm{GaAs}$ buried heterostructure laser fabrication process," Japanese Journal of Applied Physics, vol. 30, no. 3, pp. 499-500, 1991.

[27] R. V. Ghita, M. F. Lazarescu, A. S. Manea, C. Logofatu, E. Vasile, and V. Ciupina, "Facet oxidation and degradation of AlGaAs/GaAs pulsed laser diodes," Proceedings of SPIE, vol. 5581, 2004.

[28] D. G. Bell, "Group theory and crystal lattices," Reviews of Modern Physics, vol. 26, no. 3, pp. 311-320, 1954.

[29] M. S. Dresselhaus, G. Dresselhaus, and A. Jorio, Group Theory-Application to the Physics of Condensed Matter, Springer, Berlin, Germany, 2008. 